\title{
Room to Improve Conjugated Polymer-Based Solar Cells: Understanding How Thermal Annealing Affects the Fullerene Component of a Bulk Heterojunction Photovoltaic Device
}

\author{
Alexander L. Ayzner, Darcy D. Wanger, Christopher J. Tassone, Sarah H. Tolbert, and \\ Benjamin J. Schwartz* \\ Department of Chemistry and Biochemistry, University of California, Los Angeles, Los Angeles, \\ California 90095-1569
}

Received: August 27, 2008; Revised Manuscript Received: October 20, 2008

\begin{abstract}
We examine how thermal annealing affects the fullerene network in conjugated polymer bulk heterojunction (BHJ) solar cells. We begin by creating electron-only devices with a BHJ geometry by blending the fullerene derivative [6,6]-phenyl-C61-butyric-acid-methyl-ester (PCBM) with polystyrene (PS). These electron-only PS:PCBM diodes function even with a poly(ethylenedioxithiophene):poly(styrenesulfonate) (PEDOT:PSS) layer, indicating that PEDOT:PSS films do not serve as electron blocking layers. Atomic force microscopy shows that the degree of phase segregation in the PS:PCBM blend films is similar to that in the active layer of blends of PCBM with poly(3-hexylthiophene-2,5-diyl) (P3HT), so that the PS:PCBM blends provide a good model for the fullerene part of the BHJ network in P3HT:PCBM solar cells. We find that thermal annealing dramatically decreases the electron current that flows in the PS:PCBM diodes, suggesting that annealing leads to increased phase segregation that lowers the electron mobility on fullerenes in the BHJ geometry. We also find that annealing increases the photoluminesence of P3HT:PCBM blend films, indicating that thermal treatment produces increased phase segregation that leads to decreased exciton harvesting. The fact that annealing decreases both exciton harvesting and electron mobility implies that there is significant room to further improve polymer/fullerene photovoltaics by controlling the amount of phase segregation.
\end{abstract}

\section{Introduction}

Thin film conjugated polymer-based photovoltaics (PVs) have become the subject of intense research as they have displayed significant potential for competition with conventional inorganicbased solar cells, reaching respectable light-to-electrical power conversion efficiencies surpassing 5\%. ${ }^{1-4}$ Although still less efficient than inorganic solar cells, polymer-based PVs are lightweight and offer substantially reduced manufacturing costs since they are amenable to inexpensive deposition techniques such as spin-coating. The most common type of polymer-based solar cell is the bulk heterojunction (BHJ) photovoltaic, which consists of an intimate blend of a conjugated polymer and an electron acceptor, usually a fullerene derivative, that form an interpenetrating network with percolated paths to both electrodes. ${ }^{5}$ The conjugated polymer serves as the light absorber: when light is incident on the device, excitons are created on the polymer chains. These excitations then migrate by means of energy transfer, generally achieving a diffusion length of $\leq 10$ nm. ${ }^{6}$ If an exciton is able to diffuse within its lifetime to an electron acceptor, it will be quenched with near unit quantum yield, creating a spatially separated electron-hole pair with the hole localized on the polymer chain and the electron on the acceptor molecule. Under the influence of the built-in electric field, these initially bound charges can then separate and migrate to the electrodes, thus producing a photocurrent.

It is well-known that BHJ device performance can be improved dramatically through postproduction thermal anneal-

\footnotetext{
* Corresponding author. E-mail: schwartz@chem.ucla.edu.
}

ing, where the device is subjected to temperatures above the polymer glass transition. ${ }^{1-3}$ Annealing is known to increase the mobility of the holes on the polymer, likely the result of strengthened electronic communication between neighboring $\pi$-systems. ${ }^{7-9}$ For example, thermal annealing of the conjugated polymer regioregular poly(3-hexylthiophene-2,5-diyl), or P3HT, leads to enhanced crystallinity and a decreased separation between adjacent polymer chains, resulting in improved charge transport. ${ }^{1,9-11}$ It is less clear, however, how annealing affects the fullerene component of a BHJ polymer solar cell: the way in which the fullerene molecules are distributed in the polymer matrix and the details of the BHJ morphology have been the subject of much debate. ${ }^{1,2,9,10,12-15}$

In this work, we directly address the question of what happens to the fullerene component of a polymer BHJ solar cell upon thermal annealing. By fabricating "fullerene-only" BHJ halfdevices (that use electronically insulating polystyrene (PS) rather than a semiconducting polymer like P3HT), we show that thermal annealing causes a decrease in the ability of the fullerene network to conduct electrons as a result of increased phase segregation of the blended polymer and fullerene components. With the aid of conjugated polymer/fullerene film fluorescence, we also show that a similar phase segregation process occurs in the active layer of BHJ photovoltaic devices fabricated from blends of $\mathrm{P} 3 \mathrm{HT}$ and the fullerene derivative [6,6]-phenyl-C61-butyric-acid-methyl-ester (PCBM; see upper right of Figure 3, below, for chemical structure) and thus that electron conduction and exciton dissociation both decrease upon thermal annealing. This implies that annealing simultaneously 
increases hole mobility in the polymer and decreases electron mobility among the fullerenes. The most "optimal" devices are thus produced when the electron and hole mobilities are balanced rather than when the polymer has the highest possible mobility. Thus, our results suggest that there is still significant room to improve the efficiency of polymer-based solar cells: if one could reverse or limit the electron mobility decrease of the fullerene network upon annealing or find methods to increase hole mobility in the polymer network without annealing, then the overall device performance could be significantly enhanced relative to the current generation of thermally annealed devices.

\section{Experimental Methods}

For PS:PCBM diodes, poly(ethylenedioxithiophene):poly(styrenesulfonate) (PEDOT:PSS, Baytron P VP A1 4083) was spin-coated at $5000 \mathrm{rpm}$ onto prepatterned indium-doped tin oxide (ITO) electrodes that had been precleaned and treated briefly with an air-plasma. The PEDOT:PSS films were then baked at $140{ }^{\circ} \mathrm{C}$ in a dry nitrogen glovebox and allowed to cool to room temperature prior to spin-coating a blended PS (10 mg/ $\mathrm{mL}, M_{\mathrm{W}}=2043000$, Aldrich, used as received) and PCBM (Nano-C $99.5 \%$ pure, used as received) solution in chlorobenzene (1:0.67 by weight PS:PCBM) at $700 \mathrm{rpm}$ (1.5 min spin time). The solution was heated for several hours at $55{ }^{\circ} \mathrm{C}$ in the dark in a nitrogen glovebox and stirred overnight. Al (40 $\mathrm{nm}$ ) was then deposited onto the films through a shadow mask under high vacuum, resulting in an active device area of 6.32 $\mathrm{cm}^{2}$. P3HT:PCBM films were made by first spin-coating PEDOT:PSS at the same conditions as above onto precleaned and plasma-treated glass substrates followed by spin-casting the active layer from a P3HT (10 mg/mL, Rieke Metals \#4002-E, used as received) and PCBM (1:1 P3HT:PCBM weight ratio) solution in chlorobenzene at $700 \mathrm{rpm}$ (1 min spin time). Both P3HT and PS blend solutions were passed through a $0.45 \mu \mathrm{m}$ PTFE filter immediately prior to spin-casting. Annealing of all spin-cast films and completed devices was done on a digitally controlled hotplate in an argon atmosphere for $20 \mathrm{~min}$. The samples were covered with a glass Petri dish during annealing and subsequently quenched by placing them onto a roomtemperature metal surface. Atomic force microscopy (AFM) was carried out using a Nanoscope V Dimension 5000 (Veeco Digital Instruments) under ambient conditions. Antimony $n$ doped silicon cantilevers (TESPW, Veeco Probes) with spring constants of $42 \mathrm{~N} / \mathrm{m}$, first longitudinal resonance frequencies between $230-410 \mathrm{kHz}$, and nominal tip radii of $8 \mathrm{~nm}$ were employed in tapping mode. Simultaneous height and phase images were acquired and reproduced from multiple samples. Current-voltage curves of our PS:PCBM diodes were measured using a Keithley model 2400 source meter. Photoluminescence spectra of P3HT:PCBM blends annealed at different temperatures were collected on a Spex Fluorolog 3 spectrofluorometer, using the same $500 \mathrm{~nm}$ excitation wavelength, integration time, and slit widths for all samples.

We note that the high molecular weight of the PS we used produced relatively thick active layers in our electron-only devices: we estimate the thickness of the active layer in our PS:PCBM diodes to be $\sim 250 \mathrm{~nm}$ based on optical absorption measurements. This is thicker than the typical $\sim 100 \mathrm{~nm}$ active layer found in most P3HT:PCBM photovoltaic devices (and in our P3HT:PCBM blend films). When we lowered the PS concentration by a factor of 2 (with the PS:PCBM ratio held fixed) to produce thinner films, the current densities in our PS: PCBM diodes were of the same order of magnitude (a few hundred $\mathrm{mA} / \mathrm{cm}^{2}$ ) as those in P3HT-based devices at the same voltage. ${ }^{16}$ The fact that the current densities in our PS:PCBM diodes are still somewhat lower than those in P3HT:PCBM diodes is likely due to the significant amount of hole current passing through $\mathrm{P} 3 \mathrm{HT}$. The fact that the current densities are comparable, however, suggests a similar fullerene network morphology in both PS and P3HT blends. In the results presented below, we chose to concentrate on the thicker PS: PCBM diodes made from the higher-concentration PS solutions because the thicker devices showed smaller batch-to-batch variations.

\section{Results and Discussion}

Despite all the interest in conjugated polymer/fullerene BHJ solar cells, the studies done to understand the morphology of the fullerene network and the changes it undergoes upon annealing have led to controversy. For example, the facts that the blend films are predominantly amorphous and that the two components have similar electron densities make the interpretation of electron and other microscopy experiments ambiguous when it comes to elucidating the details of the blend morphology $;^{5,9,12-14}$ such techniques are also unable to yield molecular information about changes in charge conduction in the course of thermal annealing. The effects of annealing on electron mobility also are difficult to extract from the current-voltage $(\mathrm{J}-\mathrm{V})$ characteristics of working P3HT:PCBM photovoltaics because there is no simple way to detangle the roles of the polymer and fullerene in the overall device current: even the use of low work function electrodes ${ }^{17}$ does not guarantee that all of the observed current is conducted solely through the fullerene network since we have observed that P3HT can serve as the active layer for polymer LEDs when the cathode is a low work function metal like calcium.

To circumvent these difficulties and answer questions about how annealing affects carrier mobility in the fullerene phase, we have fabricated fullerene-only BHJ diodes that are functionally identical to working BHJ photovoltaic devices except that the semiconducting polymer is replaced with polystyrene (PS), an electrically inert yet chemically similar polymer to $\mathrm{P} 3 \mathrm{HT}$. To the extent that the fullerene distribution is similar when blended with PS or P3HT, monitoring the changes in the injected current in PS:fullerene devices allows us to investigate the effects of annealing on electronic conduction in the fullerene phase of BHJ photovoltaics. Previous studies have examined charge transport in vapor-deposited ${ }^{18}$ and spin-cast ${ }^{19}$ films as well as microcrystalline powders ${ }^{20}$ of pure fullerene derivatives, but to our knowledge, there are no studies examining how transport properties change upon annealing in the isolated fullerene network of bulk heterojunction PV devices. Thus, we fabricated PS:PCBM blend films because PCBM is known to produce the most efficient BHJ solar cells when used in combination with P3HT. ${ }^{1-3}$ We chose a 1:0.67 PS:PCBM weight ratio since it provides the molar equivalent of a $\sim 1: 1$ P3HT:PCBM weight ratio, which produces near-optimal P3HT: PCBM devices. ${ }^{2}$

Before examining the device behavior of our PS:PCBM diodes, we must first establish that our PS:PCBM films have a nanometer-scale morphology similar to the P3HT:PCBM films used in functioning solar cells so that any comparisons we make will be valid. The use of PS matrices for testing the carrier mobility of electron-transporting phases in organic devices has precedent in the recent work of Keivanidis et al., who studied phase segregation-induced changes in the photophysical properties of perylene tetracarboxylic diimide upon thermal annealing and found that the phase segregation properties of this organic 

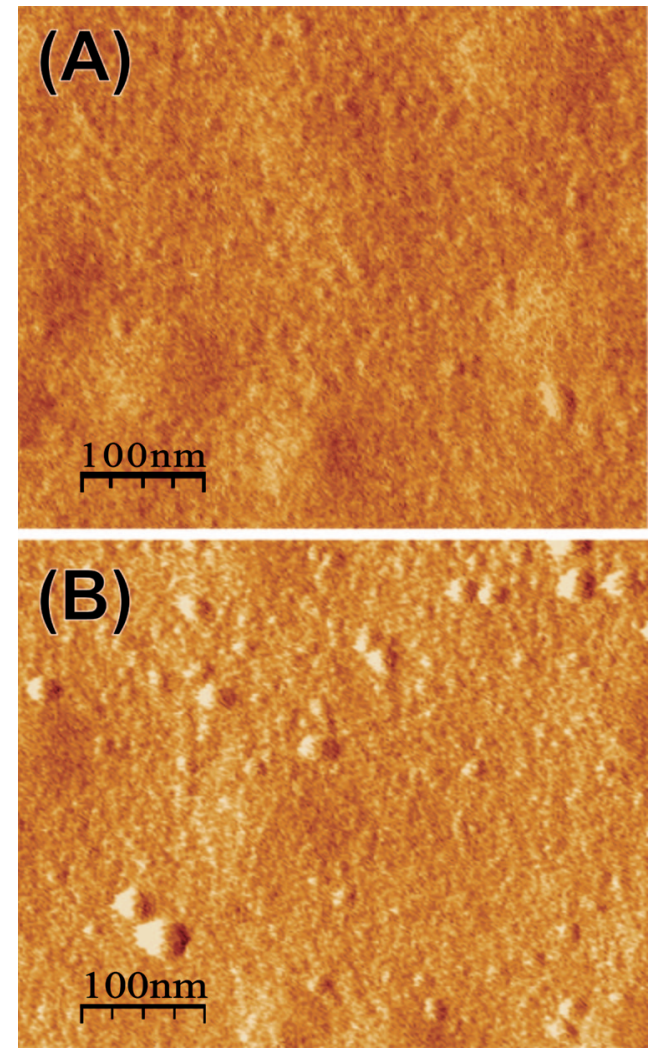

Figure 1. AFM tapping mode phase images of the surface of a 1:0.67 PS:PCBM film both (A) as-cast and (B) after thermal annealing for 20 min. In both images, a phase-separated morphology with nanometerscale domains is clearly observed. The phase-separated domains are observed to grow from $\sim 7 \mathrm{~nm}$ to $\sim 12 \mathrm{~nm}$ in diameter upon thermal annealing, suggesting that annealing increases the aggregation of PCBM in the film. Although the domain sizes are not identical, the overall phase-separated morphology and behavior upon annealing is very similar to that of P3HT:PCBM blend films (see text), indicating that PS:PCBM blend films provide a reasonable model for studying conduction in the fullerene portion of polymer-based bulk heterojunction solar cells.

molecule in inert PS matrices are similar to those in electrically active polymers. ${ }^{21}$ To verify that that the nanometer-scale morphology of PS:PCBM and P3HT:PCBM blend films is similar, we chose to use atomic force microscopy (AFM). Although unambiguous assignment of the features observed in organic PV scanning probe microscopy images has been notoriously difficult, P3HT:PCBM blend films are known to have a phase-separated morphology with nanometer-scale domains that are easily visible in the film surface topography.,12,13 Moreover, recent work examining the cross-sectional topography of P3HT:PCBM films suggests that the surface topography of such films correlates relatively well with the bulk network morphology. ${ }^{13}$

Figures 1A shows an AFM phase image of an as-cast 1:0.67 PS:PCBM blend film. The image shows nanometer-scale features on top of a nearly smooth film background. These features likely represent domains of PCBM that have phase separated from the PS given that pure PS films show no such features, as both we and others ${ }^{22,23}$ have observed. The diameter of these domains is $\sim 7 \mathrm{~nm}$, which is very similar to the size of the analogous features seen via AFM in P3HT:PCBM films. ${ }^{13}$ Figure 1B shows an AFM phase image of a 1:0.67 PS:PCBM film after thermal annealing at $110{ }^{\circ} \mathrm{C}$ for $20 \mathrm{~min}$. We chose this annealing condition since it matches closely ${ }^{24}$ with the conditions used to enhance the performance of P3HT:PCBM

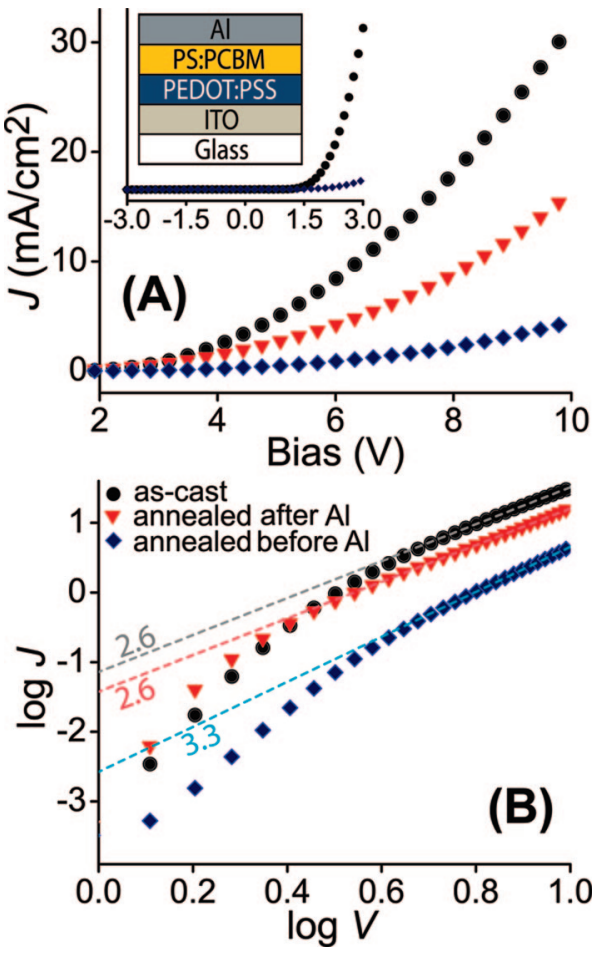

Figure 2. Current density of PS:PCBM diodes as a function of applied bias for as-cast blend films (black circles), blend films annealed after deposition of the $\mathrm{Al}$ cathode (red triangles), and blend films annealed before $\mathrm{Al}$ deposition (blue diamonds) plotted on both linear (panel A) and logarithmic (panel B) scales. The dashed curves in panel B are linear fits to the data at higher voltages with the indicated slopes. The inset shows the curves to negative bias as well as the device architecture, which was chosen to match that of P3HT-based polymer photovoltaics. The current decrease indicates that the phase segregation that occurs upon annealing detrimentally affects carrier transport in the fullerene half of the bulk heterojunction network.

solar cells. ${ }^{1-3}$ A comparison of Figures 1A and 1B makes clear that annealing PS:PCBM films increases the size of the domains to $\sim 12 \mathrm{~nm}$ in diameter. The changes in the phase-separated regions observed here strongly resemble the changes in film morphology that P3HT:PCBM blends have been observed to undergo upon thermal annealing: for both PS:PCBM and P3HT: PCBM blend films, the domains become similarly more distinct upon annealing, and the size of the domains increases in a comparable way upon annealing. ${ }^{1,13,25,26}$ Thus, based on these results, we conclude that even though the nanometer-scale domain structures are not identical, PS:PCBM blends should provide a good platform to extract trends regarding charge transport of the fullerene component of P3HT:PCBM solar cells.

Now that we have evidence that the fullerene network morphology is similar when PCBM is blended with P3HT or PS, we can use the behavior of diodes based on PS:PCBM blend films to study how thermal annealing affects electron transport on the PCBM fullerene network in a BHJ-like geometry. Our PS-based diodes were made using procedures and architecture identical to those used to make P3HT-based solar cells (see Figure 2, inset), with the active film layers prepared using the same conditions as for the AFM studies shown in Figure 1. The black circles in Figure 2A show the typical current-voltage $(\mathrm{J}-\mathrm{V})$ characteristics of our PS:PCBM diodes. The devices are strongly rectifying (see inset) and turn on around $+1.5 \mathrm{~V}$ forward bias, consistent with the expected behavior of an electron-only diode whose charge carriers are injected only from the $\mathrm{Al}$ cathode. The diodes show no significant degradation upon consecutive applications of voltage and display current densities 

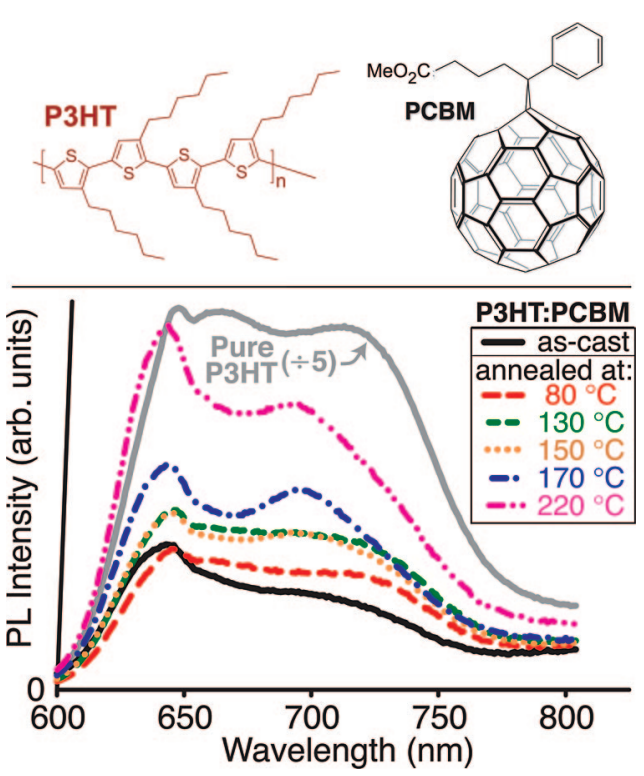

Figure 3. Upper panel: Chemical structures of P3HT (left) and PCBM (right). Lower panel: Change in photoluminescence (PL) quenching of 1:1 w/w P3HT:PCBM blend films upon thermal annealing. The PL intensities have been normalized by the optical density at the excitation wavelength. The PL intensity of a pure P3HT film (gray solid curve, $\div 5$ ) is $94 \%$ quenched by PCBM (black solid curve). The degree of quenching, however, decreases if the blend films are annealed for 20 min at different temperatures (various colored dotted and dashed curves); thus, the phase segregation that occurs upon annealing leads to decreased exciton harvesting (e.g., the $240 \%$ integrated PL increase following annealing at $220^{\circ} \mathrm{C}$ means that at most only $84 \%$ of the PL is quenched instead of $94 \%$ in the as-cast blend).

greater than $1 \mathrm{~mA} / \mathrm{cm}^{2}$ at $3 \mathrm{~V}$, indicating that the fullerenes form a percolated interpenetrating network through PS that spans the length of the film. The injected current density is comparable to that in P3HT:PCBM devices of similar thickness, which is consistent with the AFM studies in Figure 1 in suggesting that the distribution of fullerenes in the PS matrix is indeed similar to that in working P3HT:PCBM solar cells.

We note that our device architecture includes the use of a PEDOT:PSS layer between the ITO anode and the active PS: PCBM layer. Though PEDOT:PSS is still routinely viewed as an electron-blocking layer, ${ }^{27,28}$ the fact that our electron-only devices show considerable current densities indicates that PEDOT:PSS does not serve as an electron-blocking layer, an observation that is consistent with recent reports. ${ }^{19}$ Nelson and co-workers have suggested that PCBM may support a hole current as well as an electron current ${ }^{19}$ we believe, however, that the majority of the current we see in these devices is due to electrons based on the fact that changing the cathode from $\mathrm{Al}$ to $\mathrm{Ca}$ does not significantly affect the observed current density. Even if the current we observe does have a significant hole component, we still know that this current depends solely on the morphology of the fullerene network since the PS host polymer is electrically inert.

To understand the effects of annealing on carrier transport in the fullerene network of our PS:PCBM diodes, we annealed several completed devices at $110{ }^{\circ} \mathrm{C}$ for $20 \mathrm{~min}$, the same annealing conditions used for the AFM studies in Figure 1. Even though thermal annealing increases the current and power conversion efficiency of P3HT:PCBM solar cells, ${ }^{1-3}$ the red triangles in Figure 2A show that annealing completed PS:PCBM devices at $110{ }^{\circ} \mathrm{C}$ causes the current to decrease by more than a factor of 2 at $10 \mathrm{~V}$. We also found that annealing at $150{ }^{\circ} \mathrm{C}$ results in an even greater drop in current (not shown). We believe that this drop in current upon annealing is the direct result of increased phase segregation of the fullerenes in the inert polymer matrix. This is because increased phase segregation leads to increased fullerene island formation, thus decreasing the number of interconnected pathways and increasing the number of "dead-ends" in the fullerene network, producing an overall lower electron mobility through the device.

Of course, the current we measure in these devices depends on the ease of carrier injection at the electrodes as well as the mobility of the carriers in the PCBM network. Since thermal annealing can improve charge injection at organic/metal electrode interfaces, the current decrease we see represents a lower limit for the loss of mobility on the fullerene network upon annealing. To examine this in more detail, we also prepared a series of devices in which the active PS:PCBM layer was annealed prior to deposition of the $\mathrm{Al}$ cathode; in this case, we found that annealing led to a current decrease of nearly an order of magnitude at $10 \mathrm{~V}$ (Figure 2A, blue diamonds). The fact that the injected current drops more when the device is annealed before deposition of the cathode indicates that annealing does improve carrier injection at the Al/PCBM interface. This is consistent with previous reports that have established that PCBM undergoes a chemical change when in contact with $\mathrm{Al} ;{ }^{29}$ this chemistry, which is beneficial for charge injection, is likely suppressed if the $\mathrm{Al}$ is deposited after the film is annealed. But more importantly, the fact that the current decreases significantly regardless of how the interface is prepared suggests that the majority of the current decrease observed upon annealing results from decreased electron mobility due to changes in the nanometer-scale morphology of the fullerene network.

To better understand the device physics underlying the drop in conductivity upon thermal annealing, we have analyzed the $\mathrm{J}-\mathrm{V}$ characteristics of our electron-only diodes. If we assume that the carrier mobility is independent of the applied field and that the device contains localized traps that are exponentially distributed in energy below the PCBM conduction band edge, then the steady-state space-charge-limited (SCL) current formalism predicts that at high applied fields the current should be proportional to the voltage raised to the power $l+1$, where $l$ $=T_{\mathrm{c}} / T$ is the ratio of the characteristic temperature $T_{\mathrm{c}}$ that determines the width of the trap distribution to the thermodynamic temperature. ${ }^{30}$ The case with $l=1$ represents trap-free SCL transport or SCL conduction in a manifold of shallow trap states, and the presence of deeper traps leads to values of $l$ that exceed unity.

Figure $2 \mathrm{~B}$ shows the same $\mathrm{J}-\mathrm{V}$ data for the PS:PCBM diodes as Figure 2A plotted on a $\log -\log$ scale. Fits to the linear regions at higher voltages, shown as the dashed lines, give slopes of 2.6 for the devices prepared as-cast and annealed after $\mathrm{Al}$ deposition and of 3.3 for the devices annealed before $\mathrm{Al}$ deposition. The increase in slope after annealing implies the creation of additional, deeper traps, presumably because of the annealing-induced phase segregation that produces dead-ends in the fullerene network. The fact that the trap distribution is narrower for devices annealed after $\mathrm{Al}$ deposition relative to those annealed before $\mathrm{Al}$ deposition is consistent with the idea that annealing in the presence of the electrode leads to a partial elimination of traps present at the cathode-organic interface. Unfortunately, the fact that the slopes are greater than 2 means that we are unable to extract carrier mobilities from our fits since this would require independent knowledge of both the effective density of states in the PCBM conduction band and the total trap density. However, we can obtain a rough estimate of the mobility by simply assuming trap-free transport and 
forcing a linear fit with a slope of 2 through the high-voltage region. If we use this fit along with a relative permittivity of 3 and a thickness of $250 \mathrm{~nm}$, we obtain an SCL mobility $\mu$ for electrons of $\sim 2 \times 10^{-6} \mathrm{~cm}^{2} \mathrm{~V}^{-1} \mathrm{~s}^{-1}, 30$ which is in good agreement with electron mobilities extracted from time-of-flight measurements on the PS:PCBM system. ${ }^{19}$ The fact that carrier mobilities on the fullerene network are significantly smaller than those in annealed P3HT films $\left(\mu \sim 10^{-4} \mathrm{~cm}^{2} \mathrm{~V}^{-1} \mathrm{~s}^{-1}\right)$ (ref 17) indicates that the fullerene network is what limits the performance of BHJ solar cells based on annealed blends of conjugated polymers and fullerene derivatives.

Because phase segregation is ubiquitous among molecularly blended polymer systems, we expect that the thermodynamic driving force responsible for phase separation upon thermal treatment of PS:PCBM diodes also will be operative in morphologically similar P3HT:PCBM polymer-based BHJ solar cells. To investigate phase segregation in P3HT:PCBM solar cells, we have taken advantage of the relatively strong photoluminescence (PL) of conjugated polymers. Even though the PL from P3HT:fullerene blends is almost entirely quenched, the small amount of PL that remains provides a useful tool for studying the evolution of the fullerene network with annealing. We prepared samples for our PL study that were identical to those in typical working BHJ solar cells, choosing conditions to match those used in optimal photovoltaic devices. ${ }^{2}$

The lower portion of Figure 3 shows the PL spectrum of an as-cast P3HT:PCBM blend film that was photoexcited at 500 $\mathrm{nm}$ (solid black curve). A comparison of the spectrally integrated PL to that of a spin-cast film of pure P3HT (solid gray curve) shows that the presence of PCBM in a $1: 1$ weight ratio with the polymer quenches $94 \%$ of the P3HT emission. This quenching of P3HT excitons is due to electron (and possibly also energy ${ }^{31-33}$ ) transfer to any PCBM molecules that lie within the exciton diffusion radius. ${ }^{6}$ The various colored curves in Figure 3 show how the PL from the P3HT:PCBM blend changes upon annealing at different temperatures. The data show clearly that the PL intensity increases (in other words, the degree of quenching decreases) upon annealing ${ }^{10,15}$ and that higher annealing temperatures lead to a greater PL increase: the integrated PL intensity increases by a factor of 1.6 following annealing at $170{ }^{\circ} \mathrm{C}$ and by a factor of 2.4 following annealing at $220{ }^{\circ} \mathrm{C}$. This increase in PL intensity indicates that fewer P3HT excitons are able to diffuse to PCBM molecules to be quenched, implying that the average distance between the polymer and fullerene phases in the blend film has increased, consistent with the idea that annealing increases the degree of phase segregation. We note that the PL increase we observe must be due to a change in the ability of excitons to be quenched by PCBM since annealing a pure conjugated polymer film leads to a decrease in PL intensity due to increased aggregation and the formation of interchain species. ${ }^{7,34}$ Since the polymer's intrinsic PL decreases upon annealing, the extent of the decrease in PL quenching in the P3HT:PCBM blend that we observe represents a lower limit.

The data in Figure 3 have important implications for solar cells fabricated from P3HT:PCBM blends. The 60\% PL increase that we observe upon annealing at $170{ }^{\circ} \mathrm{C}$ (a temperature close to the one typically used to produce efficient devices $)^{1-3}$ means that at most $89 \%$ of the photogenerated excitons in such cells undergo charge separation, even though $94 \%$ of the excitons could have been split if the blend was not annealed. We also see that annealing at $170{ }^{\circ} \mathrm{C}$ leads to the development of enhanced vibronic structure in the PL spectrum, which has been assigned by others as being indicative of the formation of aggregated domains in P3HT microcrystals. ${ }^{11}$ The stronger interchain contacts in microcrystalline P3HT are known to produce a higher hole mobility than in the amorphous, as-cast polymer. ${ }^{8,9}$ Thus, annealing P3HT:PCBM blend films does improve hole mobility, but it also leads to increased average separation between the polymer chains and fullerene molecules, resulting in decreased exciton quenching. Like the AFM studies in Figure 1, this decrease of the PL quenching strongly suggests that the phase segregation induced upon annealing is similar in blends of P3HT:PCBM and PS:PCBM. We therefore expect that this increased phase segregation following annealing is accompanied by a drop in the electron mobility within the fullerene network of BHJ photovoltaics, just as we saw following annealing of PS:PCBM diodes.

\section{Conclusions}

In summary, Figures 1 to 3 provide direct evidence that thermal annealing detrimentally alters the nature of the fullerene network in polymer/fullerene BHJ devices. Heating a polymerfullerene blend film above the polymer's glass transition temperature may enhance the hole mobility of semiconducting polymers such as $\mathrm{P} 3 \mathrm{HT}$, but it also leads to greatly enhanced phase segregation of the two components (Figure 1). The enhanced phase segregation leads to decreased exciton splitting (Figure 3), directly lowering the maximum possible photovoltaic efficiency. Moreover, the motion of PCBM molecules to form larger crystallites or islands increases the average distance between the polymer and fullerene molecules and leads to the destruction of some of the fully percolated pathways in the fullerene portion of the BHJ network. This results in decreased electron mobility and thus decreased current (Figure 2): carriers that get stuck on islands or other dead-ends do not contribute to the bulk current.

These results have important implications for engineering the next generation of polymer-based BHJ solar cells. If the power conversion efficiency of such cells is to be further improved, it is critical to simultaneously maximize exciton harvesting and the mobilities of both the electron- and hole-transporting portions of the interpenetrating BHJ network. Even though thermal annealing improves the mobility of holes in the polymer network of a BHJ solar cell, our results show clearly that it also simultaneously decreases both exciton harvesting and the mobility of electrons in the fullerene network. Thus, it appears that the reason the power conversion efficiency of a BHJ photovoltaic increases upon annealing is that overall charge transport becomes more balanced: the initial hole mobility of polymers such as P3HT $\left(\sim 10^{-7} \mathrm{~cm}^{2} \mathrm{~V}^{-1} \mathrm{~s}^{-1}\right)$ (ref 17) is poor relative to the initial electron mobility on fullerenes like PCBM, so that the improvement in hole mobility upon annealing more than compensates for the decreases in electron mobility and exciton harvesting. Our results also explain why overannealing a BHJ solar cell can reduce power conversion efficiency: ${ }^{1}$ annealing at too high a temperature or for too long leads to an even greater destruction of the fullerene network, so that the fullerene component of the $\mathrm{BHJ}$ becomes performance-limiting because of a lower mobility and/or lower degree of exciton harvesting.

This understanding of the detrimental effects of postproduction thermal annealing on the fullerene network offers hope for the future of polymer-based photovoltaics since it indicates that there are still many avenues to explore for improving the efficiency of organic BHJ solar cells. Our results suggest that the present generation of thermally annealed polymer solar cells is efficiency-limited by our inability to simultaneously optimize 
both the polymer network and the fullerene network, whose performance decreases upon annealing. Thus, if the hole mobility of the polymer network can be improved while maintaining the exciton harvesting and electron mobility of the initial, as-cast fullerene network, then the fullerene network would no longer be performance limiting (or would be limiting at a much higher level of performance). We believe that this is the reason that alternate processing methods are starting to show great promise for the fabrication of polymer BHJ solar cells: techniques such as solvent annealing, ${ }^{35}$ slow solvent growth, ${ }^{2}$ and the use of solvent additives that preferentially dissolve the fullerene ${ }^{4}$ may provide the needed increase in hole mobility in the polymer phase without the loss of exciton harvesting and decreased electron mobility in the fullerene phase that comes upon thermal treatment. It also may be possible to improve device efficiency by chemically modifying the fullerenes to control aggregation so that annealing no longer leads to undesirable phase segregation. We will explore such an approach in future work. ${ }^{36}$

Acknowledgment. This work was supported by the National Science Foundation under Grant No. CHE-0527015 and by the Office of Naval Research under contract N-00014-04-1-0410.

\section{References and Notes}

(1) Ma, W.; Yang, C.; Gong, X.; Lee, K.; Heeger, A. J. Adv. Funct. Mater. 2005, 15, 1617.

(2) Li, G.; Shrotriya, W.; Huang, J.; Tao, Y.; Moriarty, T.; Emery, K.; Yang, Y. Nat. Mater. 2005, 4, 864.

(3) Kim, K.; Liu, J.; Namboothiry, M. A. G.; Carroll, D. L. Appl. Phys. Lett. 2007, 90, 163511.

(4) Lee, J. K.; Ma, W. L.; Brabec, C. J.; Yuen, J.; Moon, J. S.; Kim, J. Y.; Lee, K.; Bazan, G. C.; Heeger, A. J. J. Am. Chem. Soc. 2008, 130, 3619 .

(5) Hoppe, H.; Sariciftci, N. S. J. Mater. Res. 2004, 19, 1924.

(6) Shaw, P. E.; Lewis, A. J.; Ruseckas, A.; Samuel, I. D. W. Proc. SPIE 2006, 6334.

(7) Nguyen, T.-Q.; Kwong, R. C.; Thompson, M. E.; Schwartz, B. J. Appl. Phys. Lett. 2000, 76, 2454.

(8) Cho, S.; Lee, K.; Yuen, J.; Wang, G.; Moses, D.; Heeger, A. J.; Surin, M.; Lazzaroni, R. J. Appl. Phys. 2006, 100, 114503.

(9) Savenije, T. J.; Kroeze, J. E.; Yang, X.; Loos, J. Adv. Funct. Mater. $2005,15,1260$.

(10) Zhokhavets, U.; Erb, T.; Hoppe, H.; Gobsch, G.; Sariciftci, N. S. Thin Solid Films 2006, 496, 679.

(11) Korovyanko, O. J.; Österbacka, R.; Jiang, X. M.; Vardeny, Z. V.; Janssen, R. A. J. Phys. Rev. B 2001, 64, 235122.
(12) Klimov, E.; Li, W.; Yang, X.; Hoffmann, G. G.; Loos, J. Macromolecules 2006, 39, 4493.

(13) Dante, M.; Peet, J.; Nguyen, T.-Q. J. Phys. Chem. C 2008, 112, 7241.

(14) Swinnen, A.; Haeldermans, I.; vande Ven, M.; D'Haen, J.; Vanhoyland, G.; Aresu, S.; D’Olieslaeger, M.; Manca, J. Adv. Func. Mater. 2006, 16, 760 .

(15) Kim, Y.; Cook, S.; Tuladhar, S. M.; Choulis, S. A.; Nelson, J.; Durrant, J. R.; Bradley, D. D. C.; Giles, M.; McCulloch, I.; Ha, C.; Ree, M. Nat. Mater. 2006, 5, 197.

(16) Chiguvare, Z.; Dyakonov, V. Phys. Rev. B 2004, 70, 235207.

(17) Mihailetchi, V. D.; Xie, H.; de Boer, B.; Koster, L. J. A.; Blom, P. W. M. Adv. Func. Mater. 2006, 16, 699.

(18) Ma, L.; Ouyang, J.; Yang, Y. Appl. Phys. Lett. 2004, 84, 4786.

(19) Tuladhar, S. M.; Poplavsky, D.; Choulis, S. A.; Durrant, J. R.; Bradley, D. D. C.; Nelson, J. Adv. Func. Mater. 2005, 15, 1171.

(20) Warman, J. M.; de Haas, M.; Anthopoulos, T. D.; de Leeuw, D. M. Adv. Mater. 2006, 18, 2294.

(21) Keivanidis, P.; Howard, I. A.; Friend, R. H. Adv. Funct. Mater. 2008, 18,1 .

(22) Mykhaylyk, T. A.; Dmitruk, N. L.; Evans, S. D.; Hamley, I. W.; Henderson, J. R. Surf. Interface Anal. 2007, 39, 575.

(23) Qian, W.; Yao, D.; Yu, F.; Xu, B.; Zhou, R.; Bao, X.; Lu, Z. Clin. Chem. 2000, 46, 1456.

(24) Our chosen annealing temperature makes sense given the slight difference in glass transition temperature of the two polymers. See, e.g.: Ellison, C. J.; Mundra, M. K.; Torkelson, J. M. Macromolecules 2005, 38, 1767

(25) Yang, X.; Los, J.; Veenstra, S. C.; Verhees, W. J. H.; Wienk, M. M.; Kroon, J. M.; Michels, M. A. J.; Janssen, R. A. J. Nano Lett. 2005, 5, 579. (26) Campoy-Quiles, M.; Ferenzi, T.; Agostinelli, T.; Etchegoin, P. G.; Kim, Y.; Anthopoulos, T. D.; Stavrinou, P. N.; Bradley, D. D. C.; Nelson, J. Nat. Mater. 2008, 7, 158.

(27) Cravino, A.; Schilinsky, P.; Brabec, C. J. Adv. Func. Mater. 2007, 17, 3906.

(28) Yan, H.; Lee, P.; Armstrong, N. R.; Graham, A.; Evmenenko, G. A.; Dutta, P.; Marks, T. J. J. Am. Chem. Soc. 2005, 127, 3172.

(29) van Gennip, W. J. H.; van Duren, J. K. J.; Thüne, P. C.; Janssen,

R. A. J.; Niemantzverdriet, J. W. J. Chem. Phys. 2002, 117, 5031.

(30) See, e.g.: Mark, P.; Helfrich, W. J. Appl. Phys. 1962, 33, 205.

(31) Cook, S.; Ohkita, H.; Durrant, J.; Kim, Y.; Benson-Smith, J. J.;

Nelson, J.; Bradley, D. D. C. Appl. Phys. Lett. 2006, 89, 101128.

(32) Bredas, J.-L.; Beljonne, D.; Coropceanu, V.; Cornil, J. Chem. Rev. 2004, 104, 4971 .

(33) Liu, Y.-X.; Summers, M. A.; Scully, S. R.; McGehee, M. D. J. Appl. Phys. 2006, 99, 093521.

(34) Nguyen, T.-Q.; Martini, I.; Liu, J.; Schwartz, B. J. J. Phys. Chem. B 2000, 104, 237.

(35) Peet, J.; Soci, C.; Coffin, R. C.; Nguyen, T.-Q.; Mikhailovsky, A.; Moses, D.; Bazan, G. C. Appl. Phys. Lett. 2006, 89, 252105.

(36) Kennedy, R.; Ayzner, A. L.; Wanger, D. D.; Day, C. T.; Halim, M.; Khan, S. I.; Tolbert, S. H.; Schwartz, B. J.; Rubin, Y. J. Am. Chem. Soc., submitted.

\section{JP8076497}

